\title{
Successful Identification of Anti-f Alloantibody in a Non-transfused Male Patient Employing ID-Micro Typing System Gel Cards®
}

Yuki Nakamura ${ }^{1}$, Toshiya Ohsawa ${ }^{1}$, Yoshiaki Furuta ${ }^{1}$, Miho Tokida ${ }^{1}$, Kayoko Ichikawa ${ }^{1}$ and Akimichi Ohsaka ${ }^{1,2,{ }^{*}}$

${ }^{1}$ Department of Transfusion Service, Juntendo University Hospital, Tokyo, Japan

${ }^{2}$ Department of Transfusion Medicine and Stem Cell Regulation, Juntendo University School of Medicine, Tokyo, Japan

*Corresponding author: Akimichi Ohsaka, M.D., Ph.D., Department of Transfusion Medicine and Stem Cell Regulation, Juntendo University School of Medicine, 2-1-1 Hongo, Bunkyo-ku, Tokyo 113-8421, Japan, Tel: +81 35802 1109; Fax: +81 33811 2724; E-mail: ohsaka@juntendo.ac.jp

Received date: Sep 13, 2015, Accepted date: Nov 04, 2015, Publication date: Nov 09, 2015

Copyright: ( $) 2015$ Nakamura Y, et al. This is an open-access article distributed under the terms of the Creative Commons Attribution License, which permits unrestricted use, distribution, and reproduction in any medium, provided the original author and source are credited.

\begin{abstract}
Anti-f alloantibody is produced by exposure to the $f(c e)$ antigen on red blood cells (RBCs), which is a compound antigen of the Rhesus (Rh) blood group, expressed when both $\mathrm{c}$ and e antigens are present in the same haplotype (in cis). Although anti-f alloantibody was first discovered in 1953 and it has rarely been detected, the results of its detection were not reported as images in previous studies. We report a case of anti-f alloantibody in a Japanese male patient without a history of blood transfusion, whose anti-f alloantibody was successfully identified using a gel microcolumn assay (GMA). Furthermore, we reviewed the transfusion records between January 2002 and December 2010 and found that a total of 799 (1.1\%) among 73,636 blood specimens were positive for irregular RBC antibody, of which anti-f alloantibody was only observed in one patient. The present case report focuses on the usefulness of GMA to preserve the results of detecting alloantibodies as images.
\end{abstract}

Keywords: f (ce) antigen; Anti-f alloantibody; cis, Rh blood group; Gel microcolumn assay

\section{Introduction}

The Rhesus (Rh) system is one of the most immunogenic and polymorphic human blood group systems. The RHCE gene encodes both $\mathrm{C} / \mathrm{c}$ and $\mathrm{E} / \mathrm{e}$ antigens on a single protein. The $\mathrm{f}(\mathrm{ce})$ antigen is a compound antigen of the Rh blood group and expressed on red blood cells (RBCs) when both $\mathrm{c}$ and e alleles of the RHCE gene are in the cis position on the Rh chromosome (in cis), e.g., DCcee $\left(\mathrm{R}_{1} \mathrm{r}\right)$ and ccee (rr), but not in trans, e.g., $\mathrm{DCcEe}\left(\mathrm{R}_{1} \mathrm{R}_{2}\right)$. Alloantibodies directed at cis product antigens have been encountered infrequently [1]. Anti-f alloantibody was first discovered in a multiply transfused 30-year-old Caucasian male who suffered from hemophilia in 1953 [2]. In general, alloantibodies in the $\mathrm{Rh}$ blood group can cause destruction of transfused RBCs and hemolytic disease of the newborn (HDN) [3]. Anti-f alloantibody has been implicated as the cause of HDN $[4,5]$ and delayed hemolytic transfusion reaction [6]. In addition, an in vivo RBC survival test of a patient with anti-f antibody demonstrated the accelerated destruction of $\mathrm{f}$ antigen-positive RBCs [7]. Cases with anti$\mathrm{f}$ alloantibody have rarely been reported in the gel column era [8], and the results of its detection were not reported as images in previous studies.

A gel microcolumn assay (GMA) is a modified serological technique and its principle is based on the use of gel to trap agglutinates. It offers advantages in terms of result stability, versatility, and the potential for automation [9]. It is noteworthy that the reactions are stable and allow for a secondary review of results for up to 24 hours after testing has been completed [9]. In this paper, we report a rare case of anti-f alloantibody in a Japanese male patient without a history of blood transfusion, whose anti-f alloantibody was successfully identified using GMA, and its detection was reported as color images.

\section{Case Report}

A 40-year-old Japanese male suffering from mitral regurgitation was hospitalized for mitral valveplasty, and he had denied previous blood transfusions. For pre-operative examination, blood samples were presented to the transfusion service in the hospital. The standard hemagglutination method was used employing ID-Micro Typing System Gel Cards ${ }^{\oplus}$ (Bio-Rad Laboratories, Tokyo, Japan). The blood type of the patient was reported to be $\mathrm{B}, \mathrm{D}+\mathrm{C}-\mathrm{E}+\mathrm{c}+\mathrm{e}-\left(\mathrm{R}_{2} \mathrm{R}_{2}\right)$, K-, Le(a$\mathrm{b}+), \mathrm{Fy}(\mathrm{a}+\mathrm{b}-), \mathrm{Jk}(\mathrm{a}-\mathrm{b}+), \mathrm{M}+\mathrm{N}+\mathrm{S}-\mathrm{s}+$, and $\mathrm{Di}(\mathrm{a}-)$. However, the patient's plasma showed pan-reactivity on reverse blood grouping by GMA, resulting in an $\mathrm{ABO}$ discrepancy (data not shown). An indirect antiglobulin test (IAT) with the two-stage papain method was performed using a three-cell panel (ID-DiaScreen) and an 11-cell panel (ID-DiaPanel, each Bio-Rad Laboratories), showing reactivity with an anti-f antibody (Figure 1). To further investigate the patient's plasma reactivity, adsorption/elution studies were carried out on RBCs with ccee $(\mathrm{rr})$, DCCee $\left(\mathrm{R}_{1} \mathrm{R}_{1}\right)$, and $\mathrm{DCcEe}\left(\mathrm{R}_{1} \mathrm{R}_{2}\right)$ phenotypes. Adsorption of antibody in the patient's plasma was only observed in the case of the ccee (rr) phenotype (Figure 2A), and the eluates of the adsorption study showed strong (3+) agglutinates with the ccee ( $\mathrm{rr}$ ) phenotype and weaker agglutinates with the Ccee (r'r) or ccEe (rr") phenotype (Figure 2B), suggesting the presence of anti-f alloantibody. Next, a comparative titration study of the patient's plasma was performed by a low ionic strength saline (LISS)-IAT, revealing that agglutinates before 2-mercaptoethanol (2-ME) treatment showed 8 titer (Figure 3A), whereas those after 2-ME treatment showed 2 titer (Figure $3 \mathrm{~B}$ ), suggesting that the patient's anti-f alloantibody was a mixture of IgM and IgG. Finally, blood samples of the patient were referred to the Tokyo Red Cross Blood Center (Dr. Makoto Uchikawa), being confirmed to show antibody specificity as an anti-f antibody. The patient had no history of previous blood transfusion, suggesting a naturally acquired anti-f alloantibody. 
Citation: Nakamura Y, Ohsawa T, Furuta Y, Tokida M, Ichikawa K, et al. (2015) Successful Identification of Anti-f Alloantibody in a Non-transfused Male Patient Employing ID-Micro Typing System Gel Cards ${ }^{\circledR}$. J Blood Disord Transfus 6: 320. doi:10.4172/2155-9864.1000320

Page 2 of 4

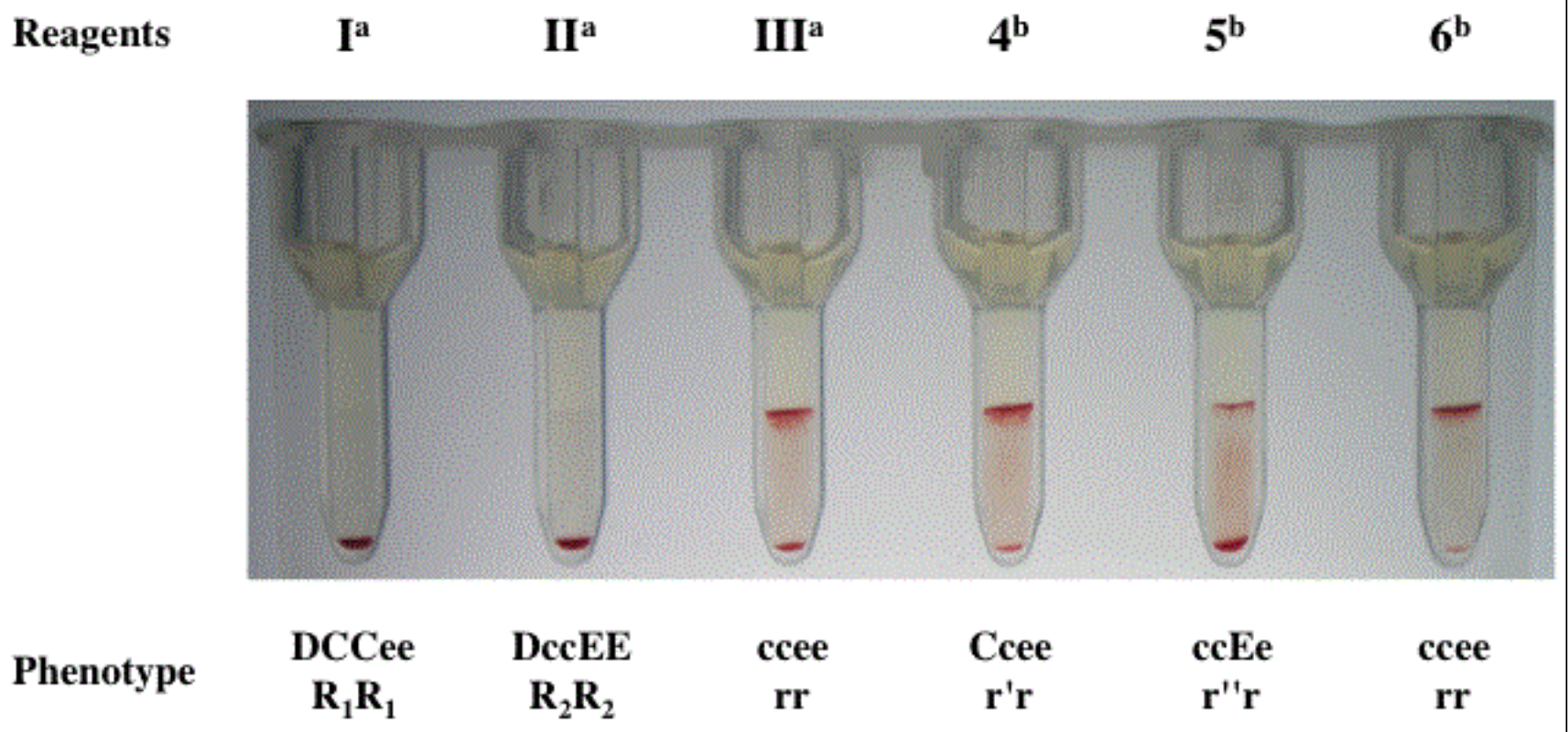

Figure 1: Identification of an alloantibody of the patient's plasma. An indirect antiglobulin test (IAT) with the two-stage papain method was performed using a three-cell panel (a) and 3 of 11-cell panel (b), showing reactivity with an anti-f antibody.

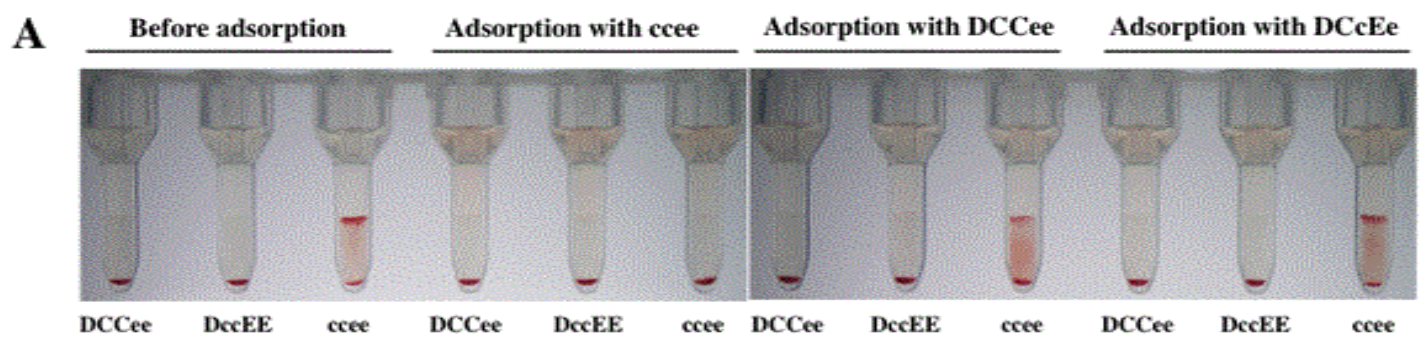

$\mathbf{B}$
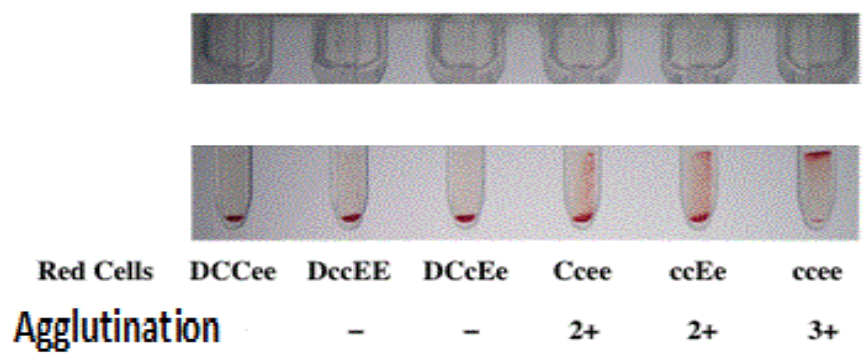

Figure 2: Adsorption/elution studies with the two-stage papain-IAT. (A) Adsorption studies were performed on RBCs with ccee (rr), DCCee $\left(R_{1} R_{1}\right)$, and DCcEe $\left(R_{1} R_{2}\right)$ phenotypes, and then the reactivity with RBCs with DCCee $\left(R_{1} R_{1}\right)$, DccEE $\left(R_{2} R_{2}\right)$, and ccee (rr) phenotypes was investigated. (B) The eluates of the adsorption studies were applied to GMA, and the reactivity with RBCs with DCCee $\left(R_{1} R_{1}\right)$, DccEE $\left(R_{2} R_{2}\right)$, DCcEe $\left(R_{1} R_{2}\right)$, Ccee $\left(r^{\prime} r\right)$, ccEe $\left(r^{\prime \prime} r\right)$, and ccee (rr) phenotypes is shown. 
Citation: Nakamura Y, Ohsawa T, Furuta Y, Tokida M, Ichikawa K, et al. (2015) Successful Identification of Anti-f Alloantibody in a Non-transfused Male Patient Employing ID-Micro Typing System Gel Cards®. J Blood Disord Transfus 6: 320. doi:10.4172/2155-9864.1000320

Page 3 of 4

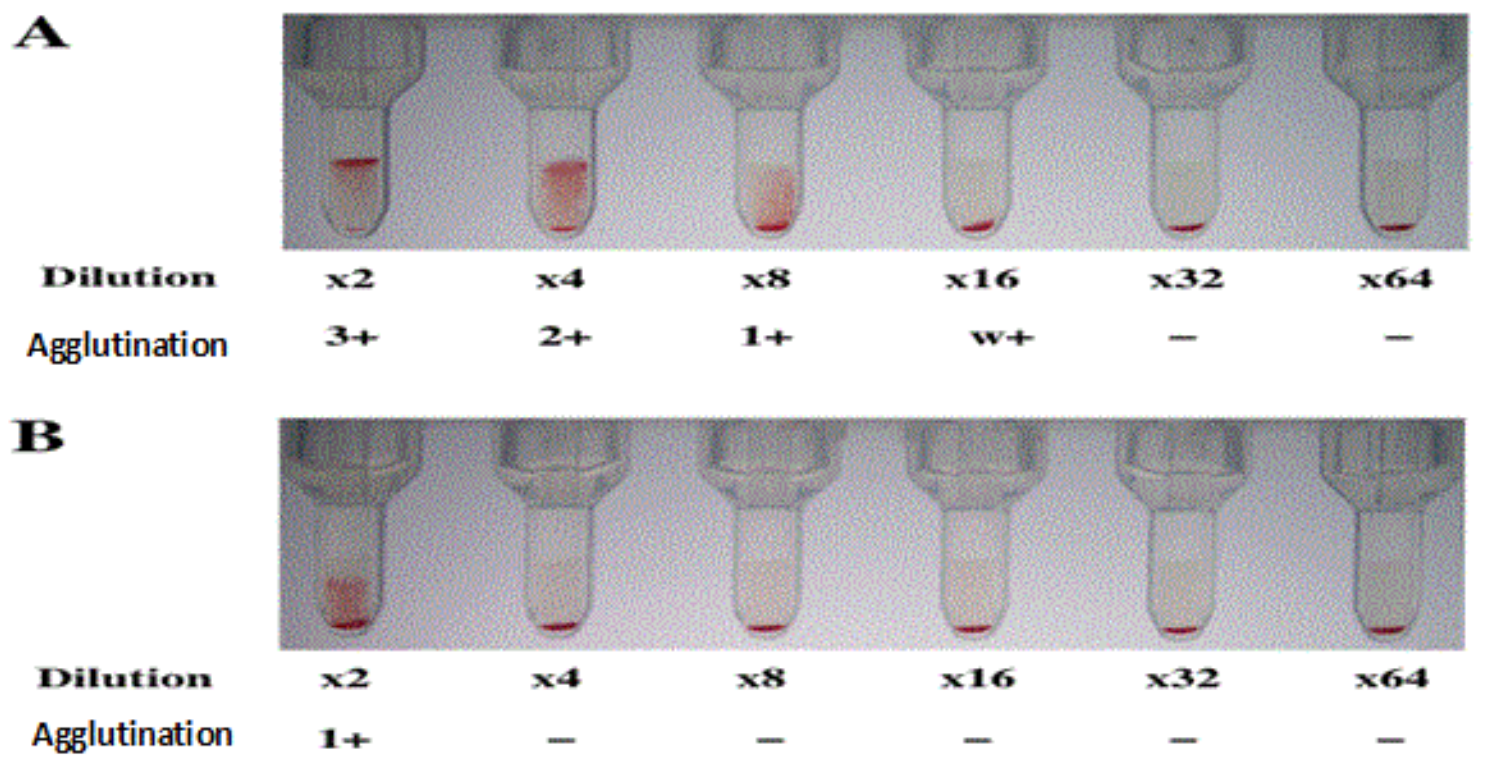

Figure 3: Comparative titration studies with a low ionic strength saline (LISS)-IAT. The patient's plasma was applied to GMA before (A) and after (B) treatment with 2-ME, showing an anti-f antibody in the patient as a mixture of IgM and IgG.

Since anti-f alloantibody has been implicated as the cause of a delayed hemolytic transfusion reaction [6], patients with anti-f alloantibody require compatible blood units, being safely transfused with units of blood lacking both $c$ and e antigens. The patient received mitral valveplasty with $800 \mathrm{~mL}$ of pre-operative autologous blood donation (PABD) and $100 \mathrm{~mL}$ of salvage blood in the operation. Although $1,000 \mathrm{~mL}$ of ce antigen-negative allogeneic blood units was reserved, the operation was completed without the need to use allogeneic blood.

\section{Discussion}

To elucidate the incidence of anti-f alloantibody, we reviewed the database of transfused patients in the hospital between January 2002 and December 2010, and found that a total of 73,636 blood specimens were screened for irregular RBC antibodies, of which 799 (1.1\%) were positive for irregular antibodies in 774 patients. In this study, an antibody being identified from the same patient during the study period was counted as one. When one (or more) different antibody was additionally detected during the study period, they were counted as two (or more). The number and rate (\%) of the identified irregular RBC antibodies were as follows: anti-E 253 (31.7), anti-Le 217 (27.2), anti-M 49 (6.1), anti-E+c 42 (5.3), anti-Cw 28 (3.5), anti-C+e 24 (3.0), anti-D 20 (2.5), anti-Le ${ }^{\mathrm{a}}+\mathrm{Le}^{\mathrm{b}} 16$ (2.0), anti-Fy 17 (2.1), anti- $\mathrm{P}_{1} 15$ (1.9), anti-Di ${ }^{\mathrm{a}} 14$ (1.8), anti-C 14 (1.8), anti-Le 13 (1.6), anti-c 11 (1.4), anti-Jk ${ }^{\mathrm{a}} 11(1.4)$, anti-S $9(1.1)$, anti-e $8(1.0)$, anti-Jra $4(0.5)$, anti-K 3 (0.4), anti-Bga 3 (0.4), anti-Jk 2 (0.3), anti-Fy 2 (0.3), anti-E $+\mathrm{Le}^{\mathrm{a}} 2(0.3)$, anti-E+Jk 2 (0.3), anti-E+Jk 2 (0.3), anti-E+Fy $2(0.3)$, anti-D+C 2 (0.3), anti-C+E 2 (0.3), 'anti-f $1(0.1)$ ', anti-N 1 (0.1), antiLW 1 (0.1), anti-Lu $1(0.1)$, anti-Xga $1(0.1)$, anti-S+P $1(0.1)$, anti-Le ${ }^{\mathrm{a}}$ $+\mathrm{P}_{1} 1(0.1)$, anti-Jk $\mathrm{j}^{\mathrm{b}}+\mathrm{Di}^{\mathrm{a}} 1(0.1)$, anti-E $+\mathrm{P}_{1} 1(0.1)$, anti-E+Le $1(0.1)$, and anti-E+S+Fy 1 (0.1). Among the irregular RBC antibodies identified during the study period, anti-f alloantibody was detected in only one patient who was presented in this paper. The rate of irregular RBC antibodies in our institution was consistent with that of a recent nationwide surveillance in Japan [10], where anti-f alloantibody was not reported. The prevalence and specificity of irregular RBC antibodies may vary among different geographic areas and races, being reported from 0.49 to $2.4 \%$ in hospitalized patients [11-16]. These studies also reported no case with anti-f alloantibody.

GMA has been used for $\mathrm{ABO}$ and $\mathrm{Rh}$ typing, the direct antiglobulin test (DAT), detecting alloantibodies, RBC phenotyping, and other applications. Although the role of GMA for DAT is controversial, GMA has been more sensitive than the conventional tube test (CTT) for detecting potentially significant antibodies coating RBCs in vivo [17]. Compared to CTT, an operator-dependent assay, GMA is less susceptible to handling errors, generates clear, more objective results, and may perform comparably to the CTT in titrating alloantibodies to $\mathrm{Rh}$ and Kell antigens [18]. In this study, we used ID-Micro Typing System Gel Cards ${ }^{\oplus}$ for detecting anti-f alloantibody in the patient, demonstrating detection as color images. Limitations of the present study are that it was a single case study and a single institutional investigation for detecting irregular RBC antibodies in hospitalized patients. Further studies are needed to establish the usefulness of GMA for preserving the results of detecting alloantibodies as images. To our knowledge, this study is the first to report the detection of anti-f antibody as color images.

\section{Conclusion}

We reported a rare case of anti-f alloantibody in a Japanese male patient without a history of blood transfusion, whose anti-f alloantibody was successfully identified by GMA. GMA may be useful for preserving the results of detecting alloantibodies as images. 
Citation: Nakamura Y, Ohsawa T, Furuta Y, Tokida M, Ichikawa K, et al. (2015) Successful Identification of Anti-f Alloantibody in a Non-transfused Male Patient Employing ID-Micro Typing System Gel Cards ${ }^{\circledR}$. J Blood Disord Transfus 6: 320. doi:10.4172/2155-9864.1000320

Page 4 of 4

\section{Acknowledgements}

We thank Dr. Makoto Uchikawa (Tokyo Red Cross Blood Center, Tokyo, Japan) for performing a confirmation study and Masahiro Ogasawara (Bio-Rad Laboratories, Tokyo, Japan) for helpful discussion.

\section{Conflict of Interests}

The authors declare no conflict of interests.

\section{References}

1. AABB Technical Manual 15th Edition (2005), Bethesda, Maryland, AABB Press.

2. Rosenfield RE, Vogel P, Sanger R, Race RR (1953) A new Rh antibody, anti-f. Br Med J 1: 975.

3. Avent ND, Reid ME (2000) The Rh blood group system: a review. Blood 95: 375-387.

4. Levine P, White J, Stroup M, Zmijewski CM, Mohn JF (1960) Haemolytic disease of the newborn probably due to anti-f. Nature 185: 188-189.

5. Spielmann W, Seidl S, von Pawel J (1974) Anti-ce (anti-f) in a CDe-cDmother, as a cause of haemolytic disease of the newborn. Vox Sang 27 473-477.

6. O'Reilly RA, Lombard CM, Azzi RL (1985) Delayed hemolytic transfusion reaction associated with $\mathrm{Rh}$ antibody anti-f: first reported case. Vox Sang 49: 336-339.

7. Ohto H, Kariyone S, Shigeta K (1989) Accelerated destruction of fpositive red cells in a patient with anti-f. Transfusion 29: 276-277.

8. Jator EK, Pedde E (2010) Anti-f in a 24-year-old male: a case study. Clin Lab Sci 23: 68-71.
9. Casina TS (2006) In search of the Holy Grail: comparison of antibody screening methods. Immunohematology 22: 196-202.

10. Takeshita A, Watanabe H, Fijihara H, Oshida M, Yurugi K, et al. (2010) Collaborative study of irregular erythrocyte antibodies in Japan: results from the Japanese study group of allo-immunity and antigen diversity in Asian populations. Transfus Apher Sci 43: 3-8.

11. Ameen R, Al-Eyaadi O, Al-Shemmari S, Chowdhury R, Al-Bashir A (2005) Frequency of red blood cell alloantibody in Kuwaiti population. Med Princ Pract 14: 230-234.

12. Tormey CA, Fisk J, Stack G (2008) Red blood cell alloantibody frequency, specificity, and properties in a population of male military veterans. Transfusion 48: 2069-2076.

13. M'baya B, Mfune T, Mogombo E, Mphalalo A, Ndhlovu D, et al. (2010) The prevalence of red cell antigens and antibodies in Malawi. Transfus Med 20: 196-199.

14. Promwong C, Siammai S, Hassarin S, Buakaew J, Yeela T, et al. (2013) Frequencies and specificities of red cell alloantibodies in the Southern Thai population. Asian J Transfus Sci 7: 16-20.

15. Xu P, Li Y, Yu H (2014) Prevalence, specificity and risk of red blood cell alloantibodies among hospitalised Hubei Han Chinese patients. Blood Transfus 12: 56-60.

16. Caamaño J, Musante E, Contreras M, Ulloa H, Reyes C, et al. (2015) Frequency and specificity of red blood cell alloimmunization in chilean transfused patients. Transfus Med Hemother 42: 4-7.

17. Novaretti MC, Jens E, Pagliarini T, Bonifacio SL, Dorlhiac-Llacer PE, et al. (2004) Comparison of conventional tube test technique and gel microcolumn assay for direct antiglobulin test: a large study. J Clin Lab Anal 18: 255-258.

18. Finck R, Lui-Deguzman C, Teng SM, Davis R, Yuan S (2013) Comparison of a gel microcolumn assay with the conventional tube test for red blood cell alloantibody titration. Transfusion 53: 811-815. 